\title{
Bimatoprost Ophthalmic Solution
}

National Cancer Institute

\section{Source}

National Cancer Institute. Bimatoprost Ophthalmic Solution. NCI Thesaurus. Code C88294.

A sterile ophthalmic solution containing $0.03 \%$ of a synthetic prostaglandin analog bimatoprost with hair-growing and anti-glaucoma activities. Applied once daily to the upper eyelid marg in at the base of the eyelashes and, optionally, to the eyebrows, bimatoprost penetrates into the hair follicle and may, through a mechanism that has yet to be fully understood, stimulate the transition of hair follicles from the telogen phase into the anagen phase and may increase the duration of the time follicles spend in anagen. By increasing the numbers of hair follicles in and duration of anagen phase, bimatoprost may help increase eyebrow and eyelash growth and appearance, including their length, thickness and darkness. 